\title{
Neue Aufgaben
}

Lösungen sind bis zum 10. Mai 2009 erbeten. Sie können auf postalischem Weg (bevorzugt) an

Dr. Hansruedi Widmer, Boldistrasse 52, Rieden, CH-5415 Nussbaumen

gesandt werden. In einem gängigen Format abgefasste Lösungen können auch als Attachment über die E-Mail-Adresse h.widmer@alumni.ethz.ch eingereicht werden.

Aufgabe 1260: Unter den natürlichen Zahlen $n$, deren Dezimalschreibweise aus 174174 Stellen besteht, betrachten wir jene mit folgender Eigenschaft: Es gibt eine natürliche Zahl $q>1$, so dass die Dezimaldarstellung von $q \cdot n$ aus jener von $n$ dadurch entsteht, dass man die letzte Ziffer an den Anfang transferiert. Wie viele solche Zahlen gibt es?

(Beispielsweise besitzt die zwölfstellige Zahl 179487179487 die gewünschte Eigenschaft, denn $4 \cdot \underline{179487179487}=7 \underline{17948717948}$.)

Panagiotis Cheilaris, Athen, GR

Aufgabe 1261: Zeige, dass es keine zweimal differenzierbare Funktion $f$ gibt, welche auf $[0, \infty)$ definiert ist und die den Ungleichungen

$$
f(x)>0 \text { für } x \geq 0, \quad f(x) \cdot f^{\prime \prime}(x)+1 \leq 0 \text { für } x \geq 0,
$$

genügt.

Vicenţiu Rădulescu, Craiova, RO

Aufgabe 1262 (Die einfache dritte Aufgabe): Zwei Gegenkanten eines Tetraeders sind orthogonal, wenn eine bestimmte Beziehung zwischen den Längen der anderen vier Kanten besteht.

a) Finde diese Beziehung.

b) Welche Höchstsymmetrie (grösste Ordnung der Symmetriegruppe) kann ein Tetraeder haben, falls es genau zwei orthogonale Gegenkanten besitzt?

Karl Wirth, Zürich, CH 


\section{Lösungen zu den Aufgaben in Heft 4, 2007}

Aufgabe 1248. Es sei $z \in \mathbb{C}$ und $n \in \mathbb{N} \quad(n \geq 2)$. Beweise:

$$
\left|1+z+z^{2}+\ldots+z^{n-1}\right|^{2} \leq\left(1+|z|^{2}+\frac{2}{n-1} \Re(z)\right)^{n-1}
$$

Mihaly Bencze, Brasov, RO

Auswertung der eingesandten Lösungen. Es sind 6 Lösungen eingetroffen, nämlich von Peter Bundschuh (Köln, D), Friedhelm Götze (Jena, D), Frieder Grupp (Schweinfurt, D), Walther Janous (Innsbruck, A), Joachim Klose (Bonn, D) und Albert Stadler (Meilen, CH).

Die einfachsten Lösungen stammen von Albert Stadler und Friedhelm Götze, die erkannt haben, dass es sich bei der zu beweisenden Ungleichung um eine Folgerung aus der Ungleichung zwischen dem geometrischen und dem arithmetischen Mittel handelt. Wir benützen folgende Beziehungen:

$$
\begin{gathered}
1+z+z^{2}+\ldots+z^{n-1}=\frac{z^{n}-1}{z-1}=\frac{\prod_{\ell=1}^{n}\left(z-e^{\frac{2 \pi i \ell}{n}}\right)}{z-1}=\prod_{\ell=1}^{n-1}\left(z-e^{\frac{2 \pi i \ell}{n}}\right), \\
\sum_{\ell=1}^{n} e^{\frac{2 \pi i \ell}{n}}=0 \Longleftrightarrow \sum_{\ell=1}^{n-1} e^{\frac{2 \pi i \ell}{n}}=-1 .
\end{gathered}
$$

Mit ihnen folgt

$$
\begin{aligned}
& \left|1+z+z^{2}+\ldots+z^{n-1}\right|^{\frac{2}{n-1}} \stackrel{(1)}{=} \prod_{\ell=1}^{n-1}\left|z-e^{\frac{2 \pi i \ell}{n}}\right|^{\frac{2}{n-1}} \\
& =\prod_{\ell=1}^{n-1}\left(1-z \cdot e^{-\frac{2 \pi i \ell}{n}}-\bar{z} \cdot e^{\frac{2 \pi i \ell}{n}}+|z|^{2}\right)^{\frac{1}{n-1}} \\
& \leq \sum_{\ell=1}^{n-1} \frac{1-z \cdot e^{-\frac{2 \pi i \ell}{n}}-\bar{z} \cdot e^{\frac{2 \pi i \ell}{n}}+|z|^{2}}{n-1} \stackrel{(2)}{=} 1+|z|^{2}+\frac{z+\bar{z}}{n-1} \\
& =1+|z|^{2}+\frac{2}{n-1} \Re(z) .
\end{aligned}
$$

In der Ungleichung zwischen dem geometrischen und dem arithmetischen Mittel besteht genau dann Gleichheit, wenn alle an der Mittelbildung beteiligten Zahlen übereinstimmen. Das ist genau dann der Fall, wenn alle $n-1$ Beträge $\left|z-e^{2 \pi i \ell / n}\right|$ gleich sind, wenn also $z$ in der komplexen Ebene von den $n-1$ Einheitswurzeln $e^{2 \pi i \ell / n}(\ell=1,2, \ldots, n-1)$ gleich weit entfernt ist. Für $n=2$ ist das trivialerweise für alle $z$ erfüllt; für $n=3$ haben genau die Punkte der reellen Achse die gewünschte Eigenschaft, und für $n \geq 4$ erfüllt einzig $z=0$ die Abstandsbedingung. 
Aufgabe 1249. Mit den Fibonacci-Zahlen $f_{1}=f_{2}=1, f_{n}=f_{n-1}+f_{n-2} \quad(n \geq 3)$ bilden wir im ebenen $(x, y)$-Gitter einen Streckenzug $S_{n}$ wie folgt: Wir starten in $(0,0)$, gehen um $f_{1}$ nach oben, um $f_{2}$ nach rechts, .., um $f_{2 n-1}$ nach oben und schliesslich um $f_{2 n}$ nach rechts. Der Streckenzug $S_{n}$, die $x$-Achse und die Parallele zur $y$-Achse durch den Endpunkt von $S_{n}$ begrenzen ein Gitterpolygon $P_{n}$. Bestimme die Anzahl $i_{n}$ der Gitterpunkte im Innern von $P_{n}$.

Jany C. Binz, Bolligen, CH

Auswertung der eingesandten Lösungen. Es sind 13 Zuschriften eingegangen, nämlich von André Ammann (Yverdon, CH), Peter Bundschuh (Köln, D), André Calame (Sauges, CH), Friedhelm Götze (Jena, D), Frieder Grupp (Schweinfurt, D), Walther Janous (Innsbruck, A), Dieter Koller (Zürich, CH), Fritz Siegerist (Küsnacht, CH), Albert Stadler (Meilen, CH), Hans Heiner Storrer (Greifensee, CH), Walter Vetsch (St. Gallen, $\mathrm{CH}$ ), Michael Vowe (Therwil, $\mathrm{CH}$ ) und Roland Wyss (Flumenthal, $\mathrm{CH}$ ).

Wir folgen Hans Heiner Storrer: Das Gitterpolygon $P_{n}$ besteht aus $n$ aneinandergefügten auf der $x$-Achse stehenden Quadraten $Q_{1}, \ldots, Q_{n}$ mit den Seitenlängen $f_{2}, f_{4}$, $f_{6}, \ldots, f_{2 n}$. Für die Anzahl $i_{n}$ der Gitterpunkte im Innern von $P_{n}$ gilt die Rekursionsformel

$$
i_{n}=i_{n-1}+\left(f_{2 n-2}-1\right)+\left(f_{2 n}-1\right)^{2}, \quad n \geq 2,
$$

mit $i_{1}=0$. Beim Anfügen des Quadrates $Q_{n}$ (mit der Seitenlänge $f_{2 n}$ ) an $P_{n-1}$ kommen nämlich die $\left(f_{2 n}-1\right)^{2}$ Punkte im Innern von $Q_{n}$ sowie die $f_{2 n-2}-1$ Punkte an der gemeinsamen Begrenzungslinie von $Q_{n-1}$ und $Q_{n}$ hinzu. Die ersten Werte sind $i_{1}=0$, $i_{2}=4, i_{3}=55$.

Eine explizitere Formel, in der allerdings immer noch die Fibonacci-Zahlen vorkommen, ist

$$
i_{n}=\frac{1}{5}\left(f_{4 n+2}-5 f_{2 n+2}+9-2 n\right), \quad n \geq 1 .
$$

Wir beweisen die Formel (4) mit Induktion. Für $n=1$ ist

$$
\frac{1}{5}\left(f_{6}-5 f_{4}+9-2\right)=\frac{1}{5}(8-5 \cdot 3+9-2)=0,
$$

was korrekt ist. Gilt nun für $n-1$, wie behauptet,

$$
i_{n-1}=\frac{1}{5}\left(f_{4 n-2}-5 f_{2 n}+9-2(n-1)\right)=\frac{1}{5}\left(f_{4 n-2}-5 f_{2 n}+11-2 n\right),
$$

so folgt auf Grund der Rekursionsformel (3):

$$
\begin{aligned}
i_{n} & =\frac{1}{5}\left(f_{4 n-2}-5 f_{2 n}+11-2 n\right)+\left(f_{2 n-2}-1\right)+\left(f_{2 n}-1\right)^{2} \\
& =\frac{1}{5}\left(f_{4 n-2}-5 f_{2 n}+11-2 n+5\left(f_{2 n-2}-1\right)+5\left(f_{2 n}-1\right)^{2}\right) \\
& =\frac{1}{5}(\underbrace{5 f_{2 n}^{2}+f_{4 n-2}+2}_{f_{4 n+2}}-5 \underbrace{\left(3 f_{2 n}-f_{2 n-2}\right)}_{f_{2 n+2}}+9-2 n) .
\end{aligned}
$$


Werden noch die bei den Unterklammerungen benützten Beziehungen

$$
\begin{aligned}
f_{2 n+2} & =3 f_{2 n}-f_{2 n-2}, \\
5 f_{2 n}^{2} & =f_{4 n+2}-f_{4 n-2}-2
\end{aligned}
$$

bewiesen, ist der Induktionsbeweis geführt.

Für den Beweis von (5) beachten wir, dass für alle $k \geq 2$ die Formel $3 f_{k}=f_{k-2}+f_{k+2}$ gilt. (Wie üblich setzt man $f_{0}=0$.) Die Formel gilt nämlich für $k=2,3$ und somit wegen der Rekursionsformel $f_{n}=f_{n-1}+f_{n-2}$ für alle $k \geq 2$. Für den Beweis von (6) benützt man zweckmässigerweise die Binet-Formel:

$$
\sqrt{5} f_{m}=\alpha^{m}-\beta^{m} \quad(m=0,1,2, \ldots) \quad \text { mit } \alpha=\frac{1+\sqrt{5}}{2}, \beta=\frac{1-\sqrt{5}}{2} .
$$

Aus ihr folgt

$$
\begin{aligned}
5 f_{m}^{2} & =\alpha^{2 m}+\beta^{2 m}-2(\alpha \beta)^{m} \\
& =\frac{1}{\sqrt{5}}(\underbrace{\left(\alpha^{2}-\alpha^{-2}\right)}_{\sqrt{5}} \alpha^{2 m}-\underbrace{\left(\beta^{2}-\beta^{-2}\right)}_{-\sqrt{5}} \beta^{2 m})-2(-1)^{m} \\
& =\frac{1}{\sqrt{5}}\left(\alpha^{2 m+2}-\beta^{2 m+2}\right)-\frac{1}{\sqrt{5}}\left(\alpha^{2 m-2}-\beta^{2 m-2}\right)-2(-1)^{m} \\
& =f_{2 m+2}-f_{2 m-2}-2(-1)^{m} .
\end{aligned}
$$

Setzt man jetzt $m=2 n$, so ist der Nachweis von (6) erbracht.

Aufgabe 1250 (Die einfache dritte Aufgabe). Gegeben seien drei Zahlenfolgen $\left(a_{n}\right)$, $\left(b_{n}\right)$ und $\left(c_{n}\right)$, welche alle einer Rekursion vom Typ $r_{n}=3 r_{n-1}-3 r_{n-2}+r_{n-3} \quad(n \geq 4)$ genügen. Ihre Anfangswerte sind $a_{1}=7, a_{2}=39, a_{3}=95, b_{1}=24, b_{2}=80, b_{3}=168$, $c_{1}=25, c_{2}=89$ und $c_{3}=193$. Zeige, dass alle Tripel $\left(a_{n}, b_{n}, c_{n}\right)$ pythagoreisch sind.

Jany C. Binz, Bolligen, $\mathrm{CH}$

Auswertung der eingesandten Lösungen. 15 Personen haben Lösungen eingesandt: Peter Bundschuh (Köln, D), André Calame (Sauges, CH), Francesco Cavalli (Verscio, $\mathrm{CH}$ ), Albert Ghenzi (Zürich, CH), Friedhelm Götze (Jena, D), Frieder Grupp (Schweinfurt, D), Walther Janous (Innsbruck, A), Dieter Koller (Zürich, CH), Miklós Lévai (Tata, HU), Fritz Siegerist (Küsnacht, CH), Albert Stadler (Meilen, CH), Hans Heiner Storrer (Greifensee, CH), Walter Vetsch (St. Gallen, CH), Michael Vowe (Therwil, CH) und Roland Wyss (Flumenthal, $\mathrm{CH}$ ).

Fast alle Einsender argumentieren wie Michael Vowe und Frieder Grupp: Das charakteristische Polynom der vorliegenden Rekursion $r_{n}=3 r_{n-1}-3 r_{n-2}+r_{n-3}$ ist $(\lambda-1)^{3}$. Nach den bekannten Methoden wird danach

$$
r_{n}=\alpha+\beta(n-1)+\gamma(n-1)^{2},
$$

wobei die $\alpha, \beta, \gamma$ geeignete Konstanten sind. 
Für $a_{1}=7, a_{2}=39, a_{3}=95$ ergeben $\operatorname{sich} \alpha=7, \beta=20, \gamma=12$ :

$$
a_{n}=7+20(n-1)+12(n-1)^{2}=12 n^{2}-4 n-1 \text {. }
$$

Für $b_{1}=24, b_{2}=80, b_{3}=168$ ergeben sich $\alpha=24, \beta=40, \gamma=16$ :

$$
b_{n}=24+40(n-1)+16(n-1)^{2}=16 n^{2}+8 n \text {. }
$$

Für $c_{1}=25, c_{2}=89, c_{3}=193$ ergeben sich $\alpha=25, \beta=44, \gamma=20$ :

$$
c_{n}=25+44(n-1)+20(n-1)^{2}=20 n^{2}+4 n+1 .
$$

Man rechnet leicht nach, dass

$$
a_{n}^{2}+b_{n}^{2}=\left(12 n^{2}-4 n-1\right)^{2}+\left(16 n^{2}+8 n\right)^{2}=\left(20 n^{2}+4 n+1\right)^{2}=c_{n}^{2}
$$

gilt; somit sind alle Tripel $\left(a_{n}, b_{n}, c_{n}\right)$ pythagoreisch.

Peter Bundschuh zeigt zusätzlich, dass alle Tripel $\left(a_{n}, b_{n}, c_{n}\right)$ primitiv sind, dass also $\operatorname{ggT}\left(a_{n}, b_{n}, c_{n}\right)=1$ für alle $n$ gilt: Ist nämlich $p$ eine $b_{n}=8 n(2 n+1)$ teilende Primzahl, so gilt entweder $p \mid 2 n$ oder $p \mid(2 n+1)$. Im ersten Fall kann $p$ evidenterweise nicht in der Zahl $a_{n}=3(2 n)^{2}-2 \cdot 2 n-1$ aufgehen; dasselbe erkennt man im zweiten Fall an der Darstellung $a_{n}=3(2 n+1)^{2}-8(2 n+1)+4$. 